\title{
Design Bases for Modulated Complexation by Electrochemistry
}

\author{
J.O. Sánchez and P.R. Tejeda * \\ Laboratorio de Ingeniería Electroquímica, Facultad de Química, Universidad Nacional \\ Autónoma de México, Ciudad Universitaria, 04510 México D.F.
}

Received 19 April 2006

\begin{abstract}
The treatment of liquid organic phases to remove undesirable compounds is currently a priority in different industries. In oil refinery, it is necessary to reduce sulphur compounds, in gasolines, to below $50 \mathrm{ppm}$. The traditional form is catalytic hydrodesulphuration; however, this system is not effective for some compounds, e.g., alkyl substituted dibenzothiophenes.

The processes for modulated complexation by electrochemistry are based on the capacity of a complexing agent, in a certain oxidation state, to form a bond with the molecule to be removed from a liquid phase. If the contaminant, the solute, is in an organic phase, the complexant must be soluble in aqueous phase. When the complexant is formed and in contact with both phases the contaminant is extracted. In a second stage, the complexing agent is oxidized (or reduced) to a state of less affinity to the contaminant, and the aqueous phase is placed in contact with a new organic phase where the contaminant will be discharged and concentrated. The complexant agent in aqueous phase is reduced (or oxidized) to its state of higher affinity for the contaminant and recirculated to the extraction stage.

In this work, we present the conditions necessary for this process to be feasible, the criteria for the selection of the complexes, the methodology for matter balances, determination of energy consumption and thermodynamic calculations, and also three examples of an organic phase: dibenzotiophene, pyridine, and methyl thioether, using as complexing agent iron tetrakis-(p-sulphonatophenyl) porphyrin.
\end{abstract}

Keywords: modulated complexation by electrochemistry, iron tetrakis(psulphonatophenyl) porphyrin, contaminants' treatment in organic solvents.

\section{Introducción}

Se ha comprobado, en escala mundial, que los petróleos crudos extraídos del subsuelo son cada vez más pesados y tienen concentraciones cada vez más altas de azufre [1]. Se han planteado alternativas para nuevos procesos de eliminación de compuestos nocivos, una de ellas es la complejación modulada por electroquímica $[2,3]$.

\footnotetext{
*Corresponding author. E-mail address: p_roquero@entropiahumana.com.
} 
Un proceso de Complejación Modulada por Electroquímica, tal como se presenta en la Fig. 1, es una estructura cíclica que permite que ciertos solutos (por ejemplo compuestos heterocíclicos con nitrógeno o azufre) sean removidos selectivamente de una fase de alimentación $y$ sean concentrados subsecuentemente en una fase receptora. El proceso pasa por una extracción reactiva en una tercera fase que contiene un agente complejante para los heterociclos. La magnitud de la interacción entre el agente complejante y el heterociclo para formar complejos, puede ser modulada controlando el estado de oxidación del agente complejante seleccionado.

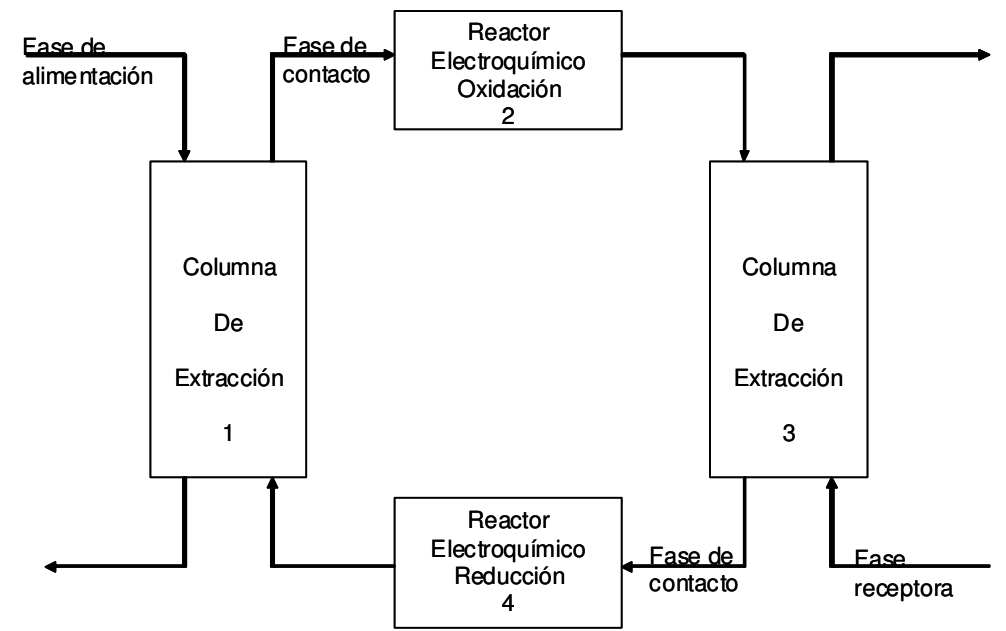

Figura 1. Diagrama de flujo de un proceso de Complejación Modulada por Electroquímica.

A continuación se presentan los cálculos termodinámicos para el diseño y evaluación de procesos de Complejación Modulada por Electroquímica. Se toma como ejemplo el caso en que la alimentación a tratar es una fase orgánica (isooctano), la fase de contacto es acuosa, conteniendo un agente complejante: la tetrakis (p-sulfonatofenil) porfirina de hierro (Fig. 2), y se analiza el comportamiento ante tres diferentes solutos: el metil tioéter, el dibenzotiofeno y la piridina.

El agente complejante debe de presentar las siguientes 4 características [4]:

1.- Debe de ser soluble únicamente en la fase de contacto (acuosa) para evitar pérdidas.

2.- Debe tener sitios de asociación y poder ser reducido y oxidado en presencia y ausencia del soluto.

3.- Debe de existir una diferencia considerable, en la afinidad por el soluto, entre los dos estados (oxidado y reducido).

4.- La cinética de la reacción entre el soluto y el agente complejante debe de ser más rápida que la transferencia de masa interfacial.

Cada etapa en el proceso continuo consiste de 4 pasos. Se puede ver en la Fig. 1 que el primer paso es poner en contacto la fase de alimentación con la fase de contacto (equipo 1); en esta etapa el agente complejante se encuentra en su estado reducido, en el que tiene gran afinidad por el soluto y se lleva a cabo una reacción de complejación agente-soluto; la formación del complejo incrementa la 
distribución del soluto en la fase de contacto, y es posible extraer el soluto de la fase de alimentación.

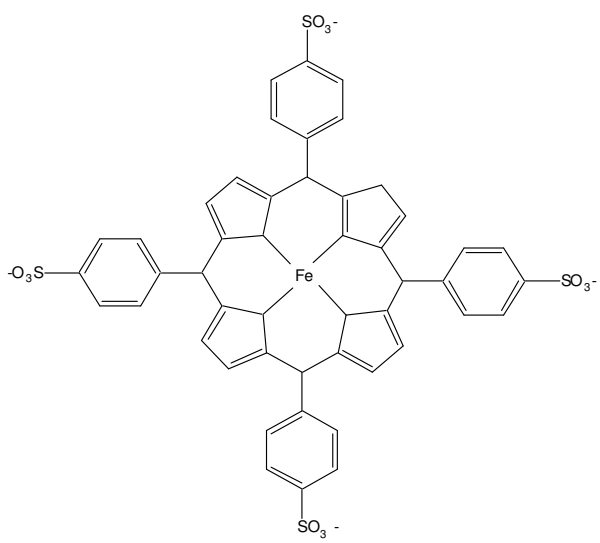

Figura 2. Tetrakis (p-sulfonatofenil) porfirina de hierro.

\section{Cálculos termodinámicos}

Una vez que se establece el equilibrio entre las dos fases, se separan, la fase de contacto es sometida a una electrólisis (equipo 2), de manera que una conversión electroquímica transforma al agente complejante a su forma de menor afinidad por el soluto (estado oxidado).

El tercer paso consiste en poner en contacto la fase acuosa con la fase receptora (equipo 3); esto hace que el soluto pase a la fase receptora y deje libre la fase acuosa. Es de esta manera que el soluto se va concentrando en la fase receptora.

El cuarto y último paso consiste en someter la fase acuosa a otra electrólisis (equipo 4), para convertir el agente complejante de nuevo a su forma reducida, terminando así un ciclo completo del proceso. Al estar de nuevo, el agente complejante, en su forma reducida, la fase de contacto puede ser recirculada al equipo 1 .

\section{a) Coeficientes de distribución}

Este proceso se basa en las siguientes reacciones de complejación que tienen lugar en la fase de contacto:

$$
\begin{aligned}
& \mathrm{C}_{\text {red }}+\mathrm{B} \rightleftarrows \mathrm{C}_{\mathrm{red}} \mathrm{B} \quad \mathrm{K}_{1} \\
& \mathrm{C}_{\mathrm{ox}}+\mathrm{B} \rightleftarrows \mathrm{C}_{\mathrm{ox}} \mathrm{B} \quad \mathrm{K}_{2}
\end{aligned}
$$

El soluto se denomina $\mathrm{B}$, mientras que $\mathrm{C}_{\text {red }} \mathrm{y} \mathrm{C}_{\mathrm{ox}}$ son el agente complejante en su forma reducida y oxidada respectivamente. $\mathrm{K}_{1} \mathrm{y} \mathrm{K}_{2}$ son las constantes de equilibrio asociadas con estas reacciones. El coeficiente de distribución $\mathrm{K}_{\mathrm{r}}$ se define como la proporción de la concentración de soluto en la fase de contacto con respecto de la que hay en la fase de alimentación cuando el agente complejante se encuentra en su forma reducida; asímismo, el coeficiente de distribución $\mathrm{K}_{\mathrm{o}}$ se define como la proporción de la concentración del soluto en la fase de contacto, con respecto de la que hay en la fase receptora cuando el agente 
complejante se encuentra en su forma oxidada. Los solutos son extraídos de la fase de alimentación disolviéndose en la fase de contacto y asociándose con el agente complejante. Cuando todo el agente complejante está en su forma reducida la concentración de soluto en la fase de contacto c después del equilibrio con la fase de alimentación es:

$$
\mathrm{c}=\mathrm{mf}+\left[\mathrm{C}_{\mathrm{red}} \mathrm{B}\right]
$$

Donde $\mathrm{m}$ es el coeficiente de distribución en la ausencia de un agente complejante y $\mathrm{f}$ es la concentración de soluto en la fase de alimentación. El segundo término de la ecuación está dado por la expresión de la constante de equilibrio de la primera reacción:

$$
\left[\mathrm{C}_{\mathrm{red}} \mathrm{B}\right]=\mathrm{K}_{1}\left[\mathrm{C}_{\mathrm{red}}\right][\mathrm{B}]=\mathrm{K}_{1}\left[\mathrm{C}_{\mathrm{red}}\right] \mathrm{mf}
$$

La concentración total del agente complejante es $\mathrm{C}_{\mathrm{T}}$. $\mathrm{X}_{\mathrm{r}}$ denota la fracción de agente complejante en forma reducida que asocia al soluto. De la definición de $\mathrm{K}_{\mathrm{r}}$ sigue que:

$$
\mathrm{K}_{\mathrm{r}}=\mathrm{m}\left[1+\mathrm{K}_{1} \mathrm{C}_{\mathrm{T}}\left(1-\mathrm{X}_{\mathrm{r}}\right)\right]
$$

Cuando el agente complejante está en la forma oxidada $\mathrm{K}_{\mathrm{o}}$ puede derivarse de la misma manera (equilibrio de las fases de contacto y receptora):

$$
\mathrm{K}_{\mathrm{o}}=\mathrm{m}\left[1+\mathrm{K}_{2} \mathrm{C}_{\mathrm{T}}\left(1-\mathrm{X}_{\mathrm{o}}\right)\right]
$$

$\mathrm{X}_{\mathrm{o}}$ es la fracción del agente complejante, en la forma oxidada, que asocia al soluto.

En este análisis se asume que ha pasado suficiente corriente, de tal manera que todo el agente complejante está siempre en su forma reducida u oxidada al tiempo en que se establece el equilibrio entre la fase de contacto y la fase de alimentación o receptora. $\mathrm{X}_{\mathrm{r}} \mathrm{y}_{\mathrm{o}}$ se han supuesto constantes cuando el número de etapas varía. De aquí que los coeficientes de distribución sean constantes durante todo el proceso. $\mathrm{K}_{2}$ es menor que $\mathrm{K}_{1}$ puesto que el agente complejante en su estado oxidado tiene menos afinidad para asociar el soluto. Para condiciones similares las ecuaciones (3) y (4) predicen que $\mathrm{K}_{\mathrm{r}}$ será mayor que $\mathrm{K}_{\mathrm{o}}$.

\section{b) Expresiones de concentración}

En este caso, se pueden repetir etapas adicionales hasta que se alcance la concentración mínima de soluto deseada en la alimentación. Después de cada etapa la expresión de la concentración en las diferentes fases puede derivarse. La concentración de la fase de contacto cambia dos veces en cada etapa. Se denomina con $c_{n} y c^{\prime}{ }_{n}$ después del primer y segundo paso en la etapa $n$ respectivamente. Así, $\mathrm{f}_{\mathrm{o}}, \mathrm{c}_{\mathrm{o}} \mathrm{y} \mathrm{r}_{\mathrm{o}}$ serán las concentraciones iniciales del soluto en la alimentación, en la fase de contacto y en la fase receptora, cada una. Después de que se establece el equilibrio en el paso $1, \mathrm{f}_{1} \mathrm{y}_{1}$ están dadas por: 


$$
\begin{array}{r}
f_{1}=\frac{f_{0}+\frac{V_{c}}{V_{f}} c_{0}}{1+\frac{V_{c}}{V_{f}} K_{r}} \\
c_{1}=K_{r} f_{1} \\
r_{1}=\frac{r_{0}+\frac{V_{c}}{V_{r}} c_{1}}{1+\frac{V_{c}}{V_{r}} K_{0}} \\
c_{1}^{\prime}=K_{0} \frac{r_{0} \frac{V_{c}}{V_{r}} c_{1}}{1+\frac{V_{c}}{V_{r}} K_{0}}
\end{array}
$$

Donde $r_{1}$ es la concentración del soluto en la fase receptora. Esto describe las concentraciones de la $1^{\mathrm{a}}$ etapa. Las relaciones del siguiente ciclo se derivan de la misma manera. En general después de $n$ etapas de equilibrio se obtienen las siguientes expresiones:

$$
\begin{gathered}
f_{n}=\frac{f_{n-1}+\frac{V_{c}}{V_{f}} c^{\prime}{ }_{n-1}}{1+\frac{V_{c}}{V_{f}} K_{r}} \\
r_{n}=\frac{r_{n-1}+\frac{V_{c}}{V_{r}} c_{n}}{1+\frac{V_{c}}{V_{r}} K_{o}} \\
K_{r}=\frac{c_{n}}{f_{n}} \\
K_{o}=\frac{c_{n}^{\prime}}{r_{n}}
\end{gathered}
$$

Un balance de masa se puede escribir en cualquier punto del proceso; su expresión al final de la etapa $\mathrm{n}$ con $\left(\mathrm{r}_{\mathrm{o}}=\mathrm{c}_{\mathrm{o}}=0\right)$ es:

$$
V_{f} f_{o}=V_{f_{n}}+V_{r} r_{n}+V_{c} c^{\prime}{ }_{n}
$$

La concentración de la fase de contacto después del equilibrio con la alimentación y la fase receptora en la etapa $n$ está dada por:

$$
c_{n}=c_{n-1}^{\prime}+\frac{V_{f}}{V_{c}}\left(f_{n-1}-f_{n}\right)
$$




$$
c_{n}^{\prime}=\frac{\frac{V_{f}}{V_{c}} f_{o}}{1+\frac{V_{r}}{V_{c}} \frac{1}{K_{o}}}-\frac{\frac{V_{f}}{V_{c}} f_{n}}{1+\frac{V_{r}}{V_{c}} \frac{1}{K_{o}}}
$$

La ecuación (14) se puede escribir como función de las concentraciones de la etapa n-1 usando la relación (9):

Si se conoce $f_{n}, c_{n}{ }_{n}$ y $r_{n}$ pueden ser determinadas usando las relaciones (12) y (15). Cuando el número de etapas se vuelve alto, la fase receptora se satura y la transferencia

$$
c_{n}=\frac{\frac{V_{c}}{V_{f}} K_{r}}{1+\frac{V_{r}}{V_{c}} K_{r}} c_{n-1}^{\prime}+\frac{\frac{V_{c}}{V_{f}} K_{r}}{1+\frac{V_{r}}{V_{c}} K_{r}} f_{n-1}
$$

de masa puede detenerse. La concentración de la fase de contacto permanece constante $\left(\mathrm{c}_{\mathrm{n}}=\mathrm{c}_{\mathrm{n}}{ }^{\prime}\right)$.

La máxima fracción de soluto que puede ser removida y la máxima fracción que puede concentrarse en la fase receptora son, respectivamente:

$$
\begin{gathered}
F_{r}=\frac{1+\frac{V_{r}}{V_{c}} \frac{1}{K_{o}}}{1+\frac{V_{r}}{V_{c}} \frac{1}{K_{o}}+\frac{V_{f}}{V_{c}} \frac{1}{K_{r}}} \\
F_{c}=\frac{\frac{V_{r}}{V_{c}} \frac{1}{K_{o}}}{1+\frac{V_{r}}{V_{c}} \frac{1}{K_{o}}+\frac{V_{f}}{V_{c}} \frac{1}{K_{r}}}
\end{gathered}
$$

La diferencia entre las dos últimas ecuaciones es la fracción del soluto que permanece en la fase de contacto. Las magnitudes de $\mathrm{K}_{\mathrm{r}} \mathrm{y} \mathrm{K}_{\mathrm{o}}$ juegan un papel importante en este tipo de separación. $\mathrm{Si} \mathrm{K}_{\mathrm{o}}$ es pequeña en comparación con $\mathrm{K}_{\mathrm{r}}$ entonces la ecuación (17) se aproxima a la unidad. Así si $\mathrm{K}_{\mathrm{o}}$ es menor que 1 entonces la ecuación (18) será cercana a 1. En otras palabras el soluto es removido de la fase de contacto y totalmente concentrado en la fase receptora. $\mathrm{K}_{\mathrm{r}} \mathrm{y} \mathrm{K}_{\mathrm{o}}$, dadas por las ecuaciones (3) y (4) son funciones de la solubilidad del soluto en la fase de contacto, la concentración total del complejante, la fracción de agente complejante que se asocia con el soluto y de las constantes de equilibrio de las reacciones.

\section{Resultados}

Las Fig. 3, 4 y 5 presentan los resultados del equilibrio líquido-líquido para los tres solutos, en ausencia de agente complejante, utilizando dos ecuaciones de 
estado (Soave-Redlich-Kwong, y Peng-Robinson), y un modelo para la energía de Gibbs de exceso (Unicuaq). Las ecuaciones de estado muestran que todos los solutos son prácticamente insolubles en agua.
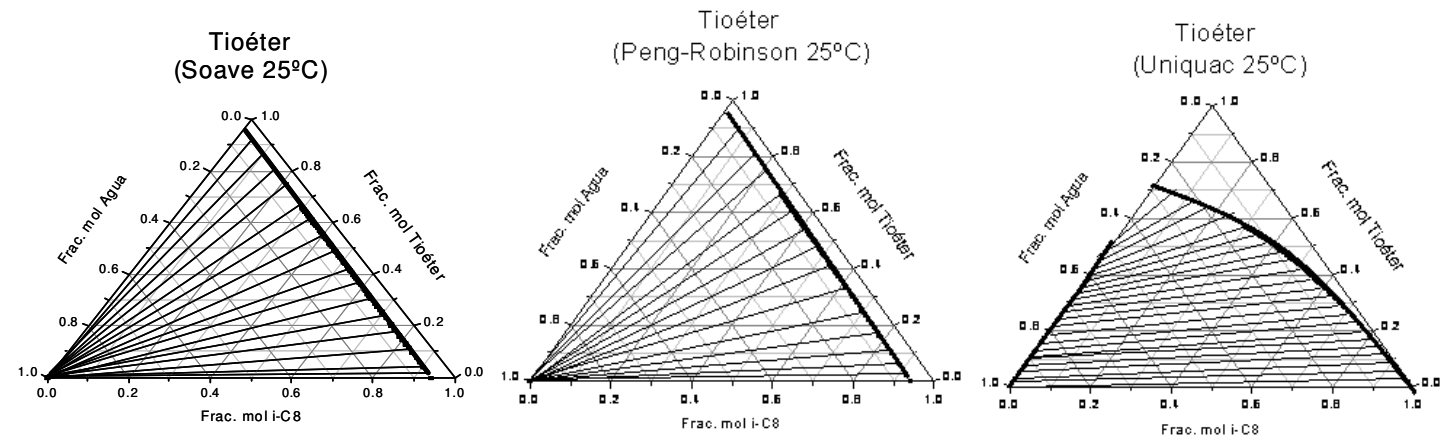

Figura 3. Cálculos de equilibrio líquido-líquido para el sistema metiltioéter / agua / isooctano, a $25^{\circ} \mathrm{C}$.
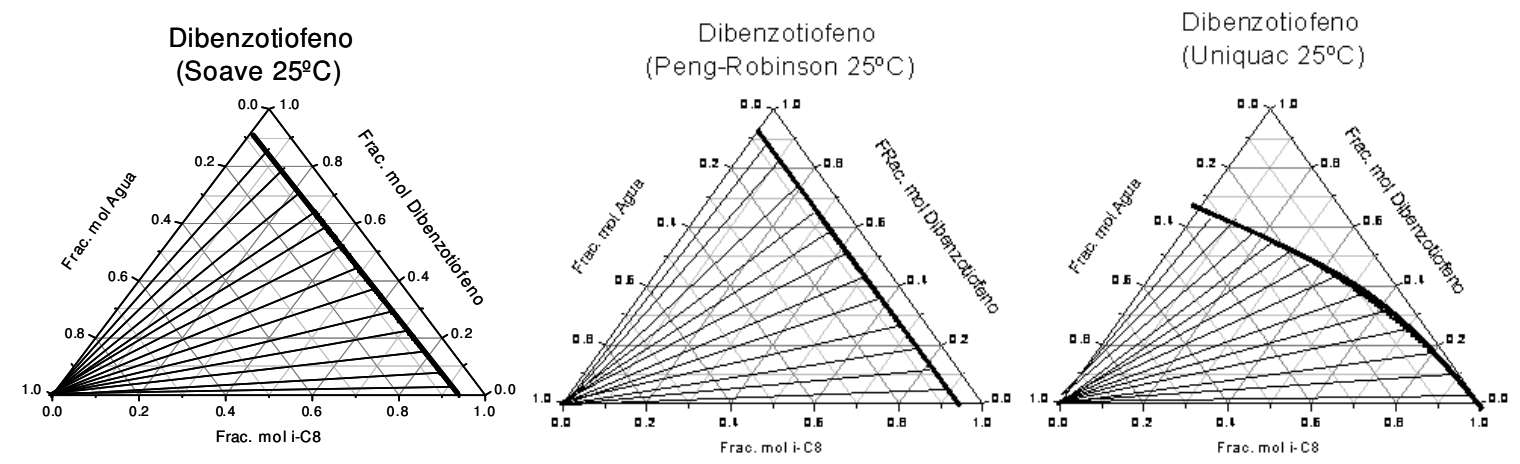

Figura 4. Cálculos de equilibrio líquido-líquido para el sistema dibenzotiofeno / agua / isooctano, a $25^{\circ} \mathrm{C}$.
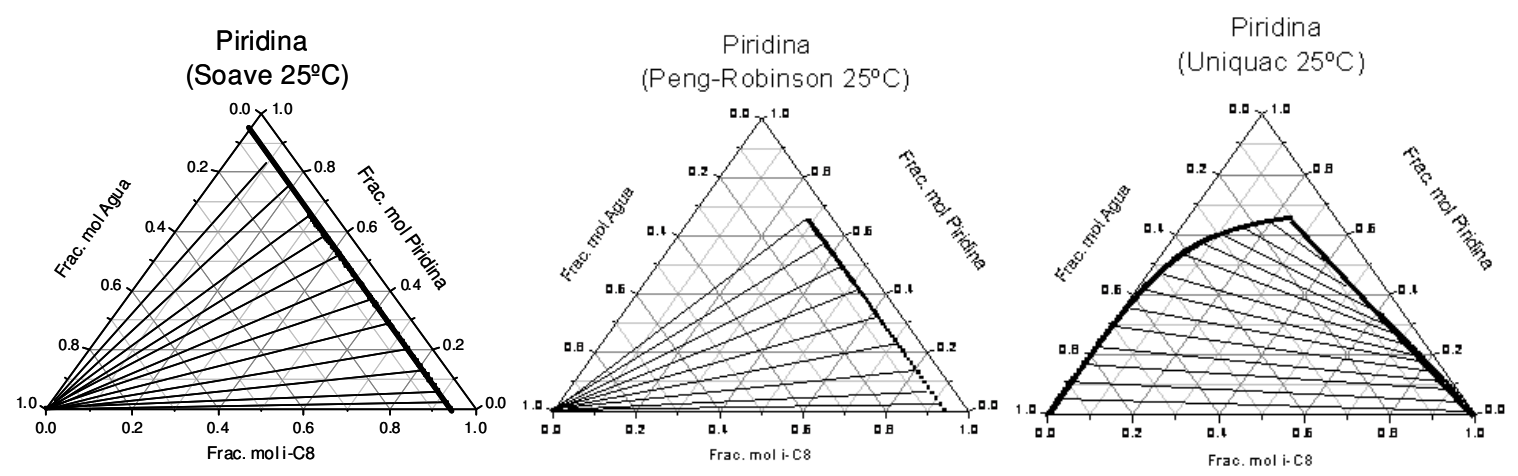

Figura 5. Cálculos de equilibrio líquido-líquido para el sistema piridina / agua / isooctano, a $25^{\circ} \mathrm{C}$.

La Fig. 6 presenta los resultados para el complejo porfirina-soluto, donde se puede observar que la pendiente de las líneas de unión cambia de signo, con respecto del puro equilibrio de fases. 

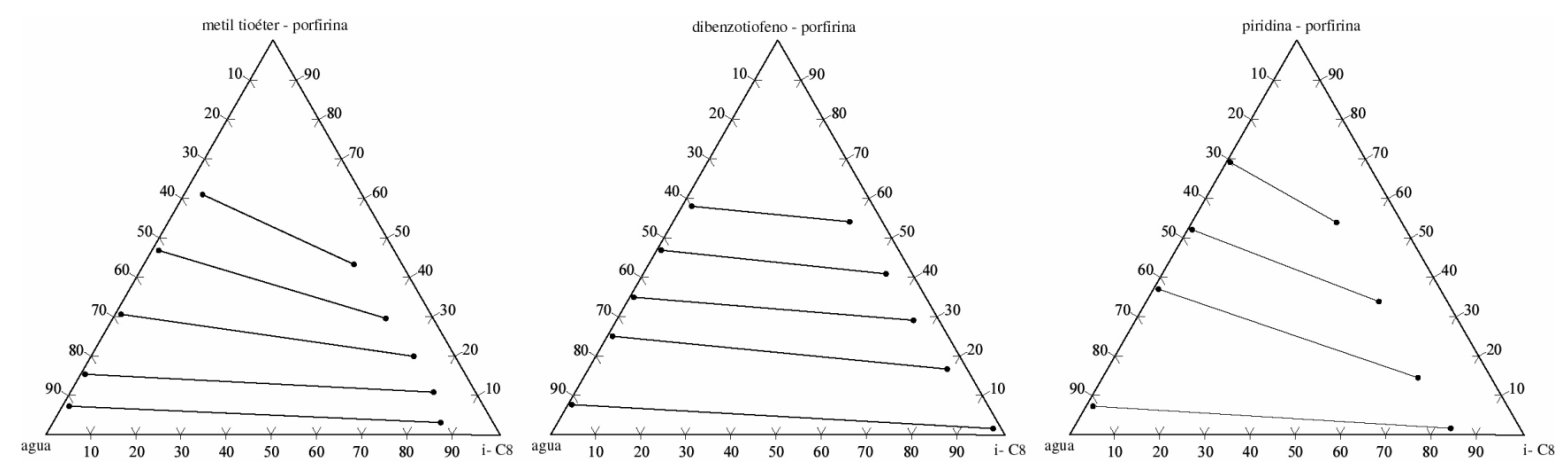

Figura 6. Cálculos de equilibrio líquido-líquido para los sistemas complejo porfirinasoluto / agua / isooctano, a $25^{\circ} \mathrm{C}$.

Las Fig. 7, 8 y 9 presentan los resultados de remoción de soluto de la fase a tratar, y su concentración en la fase receptora, mediante el análisis presentado por etapas de equilibrio. Se puede observar que para el metil tioéter y para la piridina, el agente complejante seleccionado permite la operación adecuada del proceso. Para el dibenzotiofeno los resultados muestran un pobre desempeño del proceso, que lo hace inadecaudo para esta separación.

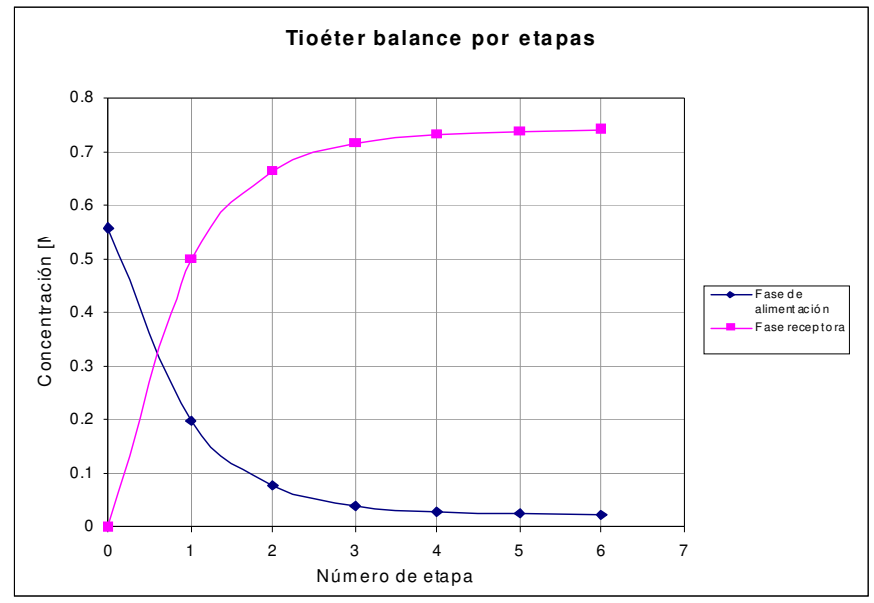

Figura 7. Resultados por etapas de equilibrio para la remoción de metil tioéter. 


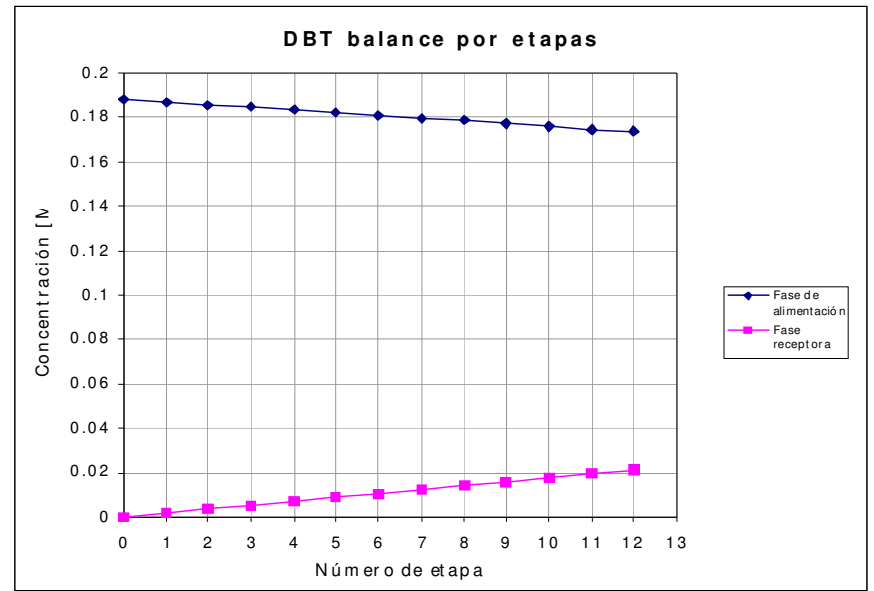

Figura 8. Resultados por etapas de equilibrio para la remoción de dibenzotiofeno.

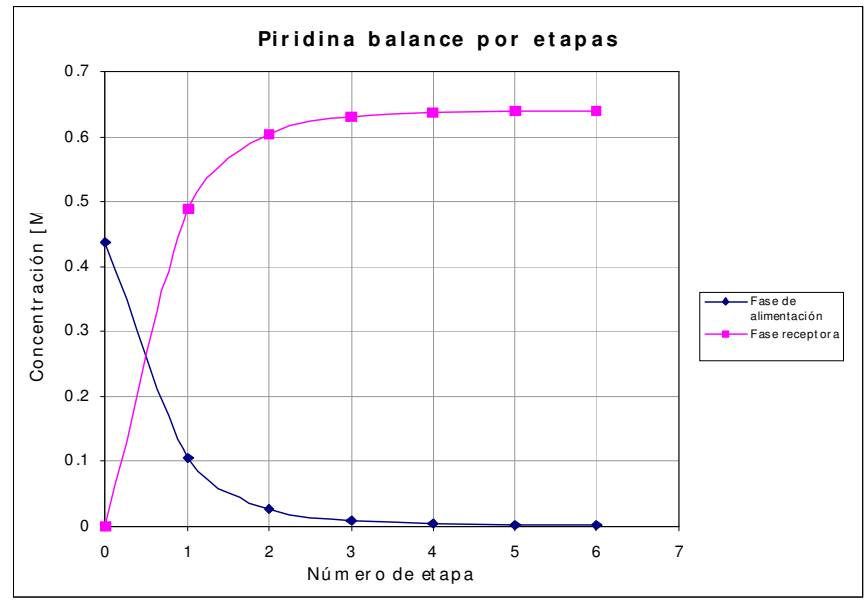

Figura 9. Resultados por etapas de equilibrio para la remoción de piridina.

\section{Conclusión}

Los procesos de complejación modulada por electroquímica son una alternativa interesante para el tratamiento de fases líquidas contaminadas. Para cada caso particular, es necesario identificar o desarrollar agentes complejantes adecuados, que puedan ser confinados en la fase de contacto, y cuyos diferentes estados de oxidación presenten una amplia diferencia de afinidad por el soluto. En este trabajo se presentan los fundamentos termodinámicos para el análisis de estos procesos. La construcción de un proceso como el descrito, requeriría el estudio previo de la cinética de las diferentes etapas involucradas. 


\section{Bases de Diseño para Procesos de Complejación Modulada por Electroquímica}

\section{Resumen}

El tratamiento de fases líquidas orgánicas para remover compuestos considerados nocivos, es actualmente una prioridad en diferentes industrias. En el caso de la refinación del petróleo, es necesario reducir, en las gasolinas, las concentraciones de compuestos que contienen azufre hasta niveles por debajo de $50 \mathrm{ppm}$. La forma tradicional de eliminar este tipo de compuesto es la hidrodesulfuración catalítica, sin embargo, existen moléculas refractarias a este tratamiento, como son los dibenzotiofenos alquil-sustituidos.

Los procesos de complejación modulada por electroquímica se basan en la capacidad de un agente complejante, en un estado de oxidación determinado, para formar un enlace con la molécula a remover de una fase líquida. Si el contaminante, o soluto, se encuentra en una fase orgánica, el agente complejante deberá ser soluble en fase acuosa. Al formarse el complejo, tras poner en contacto ambas fases, el contaminante es extraído de la fase orgánica. En una segunda etapa, el agente complejante es oxidado (o reducido) a un estado de menor afinidad por el contaminante, y la fase acuosa es puesta en contacto con una nueva fase orgánica, donde será descargado y concentrado dicho contaminante. El agente complejante en fase acuosa es reducido (u oxidado) a su estado de mayor afinidad por el contaminante y recirculado a la etapa de extracción.

En este trabajo se presentan los requerimientos para que un proceso de este tipo sea viable, los criterios de selección de complejantes, las metodologías para establecer balances de materia, determinar consumo de energía y realizar los cálculos termodinámicos. así como ejemplos para tres contaminantes de una fase orgánica: dibenzotifeno, piridina y metil tioéter, utilizando como agente complejante la tetrakis(p-sulfonatofenil) porfirina de hierro

Palabras Clave: Complejación Modulada por Electroquímica, tetrakis(psulfonatofenil) porfirina de hierro, Tratamiento de contaminantes en solventes orgánicos.

\section{Referencias}

1. R.B. Long, J.G. Speight, en Petroleum Chemistry and Refining, J.G. Speight, Editor, Taylor and Francis, 1998.

2. C.A. Koval, S.M. Drew, T. Spontarelli, R.D. Noble, Sep. Sci. and Technology 23 (1988) 1389-1399.

3. C.A. Koval, S.M. Drew, R.D. Noble, J. Yu, Inorganic Chemistry 29 (1990) 4708-4714.

4. N. Jemaa, R.D. Noble, C.A. Koval, J. Yu, Chem. Eng. Sci. 46 (1991) 10171026. 